\title{
A formação de educadores em saúde da família: uma agenda para atividades futuras em Odontologia
}

Luciana Coutinho Simões Wanderley*; Fernanda Campos Sousa de Almeida**; Cilene Rennó Junqueira***; Mariana Gabriel****; Maria Ercilia de Araujo ${ }^{* * * * *}$

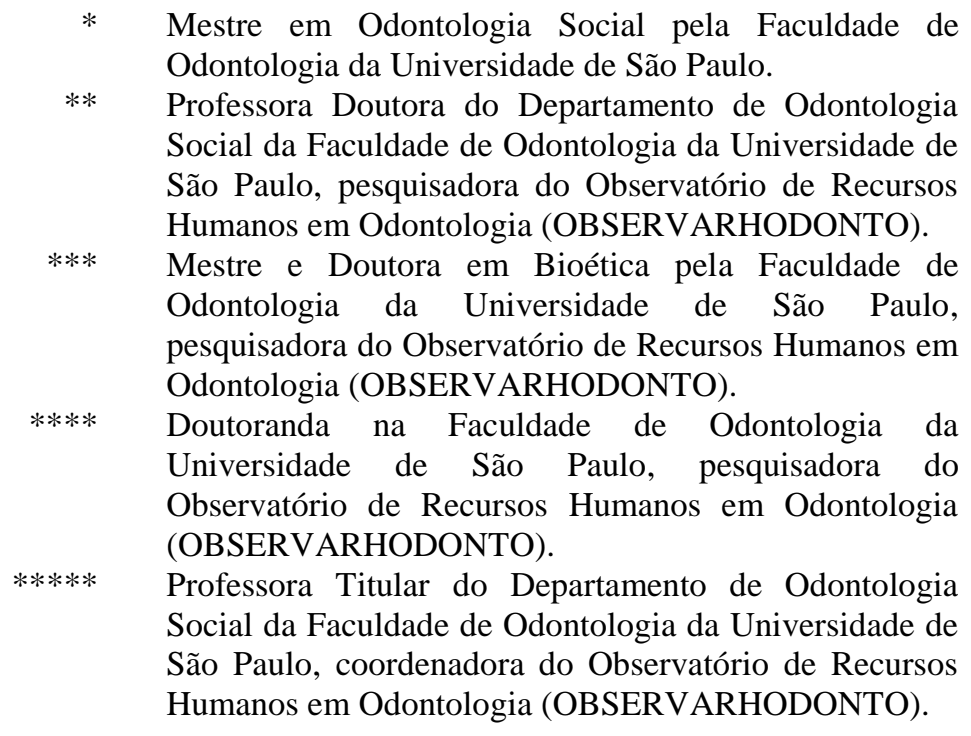

\section{RESUMO}

A residência multiprofissional é uma modalidade de pós-graduação que agrega graduados de diversas áreas da saúde e que visa a consonância da formação com as necessidades do serviço público, tendo como cenário de prática a atenção primária, principalmente por meio da Estratégia de Saúde da Família. Este estudo tem como objetivo analisar a percepção de cirurgiões-dentistas, egressos da residência multiprofissional, sobre a atuação de dois tipos de educadores desse processo: os tutores e preceptores. O estudo, de abordagem qualitativa, constituiu-se em duas etapas: a análise documental do curso da Residência Multiprofissional da Faculdade Santa Marcelina de São Paulo e a realização de entrevistas semiestruturadas com os cirurgiões-dentistas egressos do curso em questão. Após a análise, foi possível concluir que o papel do tutor e do preceptor não estava bem definido no processo de formação dos residentes, situação que dificulta a atuação dos mesmos na prática do serviço. Tornase evidente a necessidade de um melhor preparo e definição do papel destes profissionais para que as práticas pedagógicas sejam operadas a contento. Da mesma forma, se evidencia a necessidade da institucionalização da formação de recursos humanos em saúde de nível multiprofissional, nas agendas das instituições de ensino superior, a fim de que se alcance o fortalecimento da proposta na atenção primária com vistas à promoção, prevenção e recuperação da saúde da população.

Descritores: Residência. Saúde da Família. Atenção Primária à Saúde. Odontologia.

\section{INTRODUÇÃO}

As discussões a respeito da promoção da saúde adquiriram proporções mundiais em 1977 durante a 30 a Reunião 
Anual da Assembleia Mundial de Saúde, quando se decidiu que a principal meta social dos governos participantes seria a “obtenção por parte de todos os cidadãos do mundo de um nível de saúde no ano 2000 que lhes permitisse levar vida social e economicamente produtiva" conhecida atualmente como "saúde para todos no ano 2000"1. Em 1986, a Carta de Ottawa para a Promoção da Saúde ou I Conferência Internacional sobre Promoção da Saúde, que aconteceu no Canadá, definiu promoção de saúde como: ..."processo de capacitação da comunidade para atuar na melhoria da sua qualidade de vida e saúde incluindo uma maior participação no controle deste processo"2.

Desde então se tornou eminente a necessidade de criação de estratégias para a organização e fortalecimento da Atenção Básica, como o primeiro nível de atenção à saúde e em sintonia com essa necessidade o Ministério da Saúde do Brasil constituiu, em 1994, o Programa de Saúde da Família (PSF). Nessa estratégia, o trabalho em equipe multiprofissional é fundamental, pois evidencia a necessidade de saberes diferentes e articulados para resolver as demandas de saúde do indivíduo e da coletividade, ou seja, são boas oportunidades para o desenvolvimento da formação colaborativa e centrada no paciente ${ }^{3}$.

Para isso, é fundamental que os recursos humanos atuantes nessa nova perspectiva sejam competentes para trabalhar com informações epidemiológicas, demográficas, econômicas, sociais e culturais da população de cada local e, sobretudo, consigam relacionar essas questões com as ações propostas em saúde.
O Programa de Residência Multiprofissional em Saúde da Família (RMSF) visa potencializar a capacidade de mudanças na formação dos profisssionais da saúde e no atual modelo de cuidado ${ }^{4}$, propondo, por meio da Estratégia de Saúde da Família, um novo perfil de trabalho, tanto para os profissionais quanto para a organização da linha do cuidado.

Este estudo tem como objetivo analisar a percepção de cirurgiõesdentistas, egressos de um curso de residência multiprofissional, sobre a atuação de dois tipos de educadores desse processo: os tutores e os preceptores.

\section{METODOLOGIA}

O presente estudo, de caráter qualitativo, contou com duas etapas de execução: a análise documental do curso de Residência Multiprofissional da Faculdade Santa Marcelina de São Paulo, por meio da leitura minuciosa do projeto político pedagógico do curso; e a realização de entrevistas semiestruturadas com os egressos. Foram realizadas entrevistas com oito egressos de onze cirurgiões-dentistas formados pelo curso no período de 2005 a 2007. As entrevistas visaram deixar os depoentes livres para relatar suas experiências, a partir de algumas perguntas norteadoras realizadas pela pesquisadora. Os depoimentos foram gravados, transcritos e categorizados com foco no objetivo do artigo e a sistemática utilizada para a análise seguiu a sequência definida pelo método de Análise do Conteúdo proposto por Bardin ${ }^{5}$.

Essa pesquisa foi aprovada pelo Comitê de Ética em Pesquisa da Faculdade de Odontologia da Universidade de São Paulo, sob o protocolo $\mathrm{n}^{\circ}$ 180/2007. 


\section{RESULTADOS E DISCUSSÃO}

A Residência Multiprofissional em Saúde da Família é uma experiência recente, que envolve profissionais que trabalham na Estratégia de Saúde da Família, como médicos, enfermeiros e cirurgiões-dentistas, entre outros. A possibilidade de trabalhar com formação de recursos humanos, no nível de pósgraduação, procurando adequar os conteúdos ministrados para atender às necessidades da população, é um grande desafio para educadores e instituições de ensino superior. Alia-se tal possibilidade à oportunidade de trabalhar com todas as categorias de profissionais da saúde de forma interdisciplinar, multidisciplinar e transversal. Tal perspectiva oferece múltiplas possibilidades, porém, também muitos desafios.

Apresentaremos a seguir, dois atores, que exercem papel de educadores nesse processo. $\mathrm{O}$ tutor, ator principal na troca de saberes no convívio diário com o residente e o preceptor, que desempenha papel de mediador das relações entre os profissionais do serviço e a equipe dos residentes.

\section{O papel do tutor de área}

$\mathrm{Na}$ língua portuguesa, tutor significa curador, preceptor, indivíduo encarregado de tutelar, proteger, defender alguém, responsável por cuidar e amparar. Uma pesquisa que analisou a relação entre os tutores e seus alunos, em um curso de graduação em enfermagem levantou que, de fato, os tutores propiciavam sensação de segurança a seus alunos ${ }^{6}$. De acordo com o projeto político pedagógico do curso de residência, o tutor de área é um profissional, no caso da Odontologia, um dentista, vinculado a uma Equipe de Saúde da Família, que tem um papel de acompanhamento diário dos residentes, a fim de ampliar a visão do educando, voltada para oportunizar a análise de possibilidade de aplicação prática do saber conquistado. Embora o contexto entre a graduação e a pós-graduação seja distinto, em ambos os casos ocorre o processo de aprendizagem, portanto a figura de um mediador parece ser essencial.

De acordo com a análise dos discursos foi possível observar que o principal papel desempenhado pelo tutor de odontologia foi o de acompanhamento clínico e direcionamento na rotina de trabalho.

"...eu tive uma tutora também que foi bem legal. $O$ que a gente precisava, ela ajudava, então a gente foi sempre crescendo" (sujeito 1).

"Mas eu acho que o que eles passaram pra gente de mais valioso foi a rotina de trabalho" (sujeito 8 ).

Uma importante abordagem apresentada é que "tutores (mentores), com sua maior experiência que o jovem que acaba de entrar, conhecem algumas 'pedras no caminho', alguns possíveis atalhos e pontos de alerta necessários"7. Já outra abordagem, denomina tutor aquele que orienta a formação de profissionais já graduados e que atuam no sistema de saúde. É a aprendizagem que acontece em ambientes de atenção primária “(...) além de competência clínica e da capacidade de ajudar a aprender a aprender, precisa ter compreensão da prática profissional em sua essência e estimular o desenvolvimento pessoal. Espera-se que ele dê conselhos, atuando também como guia e modelo" 8 .

Uma pesquisa que avaliou a vivência do enfermeiro tutor em um hospital de especialidades oncológicas 
mostrou o auxílio dado pelo enfermeiro tutor na promoção do ensino para o desenvolvimento da sua capacitação técnica ${ }^{9}$.

Embora a literatura reconheça a importância do tutor no processo de aprendizagem do aluno, a fala dos entrevistados revelou as falhas na formação dos tutores para realização plena deste papel.

"Deixou muito a desejar, então eu acho que eles não prepararam os tutores pra gente, não teve nenhuma capacitação, nada a gente chegou lá... quem é você? Então no começo assim eles ficavam tudo meio perdido, a gente meio perdido, os tutores não sabiam muito bem o que fazer com a gente" (sujeito 1).

"Acho que a única coisa que poderia melhorar na residência mesmo... é a questão da formação do tutor (...) porque ele é um dentista que tá lá na unidade, foi contratado para ser dentista, não foi contratado para ser tutor dos residentes. Então acho, que quando tem a residência tem que ter essa estrutura da formação dos tutores pra poder receber os residentes $e$ acompanhar também a equipe multiprofissional o tutor também tem que interagir com a equipe toda, não é só com um de nós dentistas (...)" (sujeito 3).

"Tem gente que gosta de ajudar, de ser tutor, mas tem gente que não tem esse perfil. Então eu acho que a coordenação deveria selecionar profissionais interessados, não só na verba mensal extra, porque já começa por ai, se uma pessoa tem interesse então é meio caminho andado" (sujeito 4).
"Na minha turma de residência os tutores dos residentes eram os profissionais que estavam no serviço e eles não tinham formação nenhuma" (sujeito 5).

Este é um ponto crucial na residência multiprofissional - a nítida necessidade de capacitação destes profissionais (tutores) que cumprem um papel de grande importância dentro deste processo de formação. "Muitas vezes é atribuído a ele o papel de modelo. Embora ele possa também exercer esse papel, o tutor deve ser na verdade um exemplo concreto do futuro para seus tutorandos (...) e deve ajudar o aluno a encontrar o seu próprio espaço" $"$, sendo importante que se observe que quando o residente perde a confiança no seu tutor o processo de aprendizagem fica comprometido.

Sendo assim, é do tutor a responsabilidade pela condução desse processo complexo de ensinoaprendizagem, o qual irá possibilitar, ou não, o alcance de todo seu potencial. As falhas na compreensão do seu papel ou mesmo a ausência desta figura podem ser determinantes nos resultados, pois nesse estudo foram encontrados problemas com relação à supervisão local com internos em medicina social ${ }^{10}$.

Para a compreensão deste processo é importante destacar os limites e possibilidades da residência levantados por um estudo realizado no mesmo local de estudo desta pesquisa, o qual mostrou que os tutores que atuaram na RMSF eram profissionais que trabalhavam nas Unidades Básicas de Saúde (UBS) e foram selecionados pelas equipes de coordenação junto com os preceptores. Estes profissionais, ao longo de sua trajetória institucional, apresentavam 
habilidades técnicas para receber $\mathrm{e}$ acompanhar a equipe de residentes, no entanto no início os tutores não tiveram nenhuma preparação para atuar como educadores, situação que desencadeou inúmeras dificuldades de relacionamento entre tutores e residentes e em algumas vezes a troca da equipe tutorial ${ }^{11}$.

Nesse processo de aprendizagem é fundamental que os tutorandos também possuam algumas características, entre elas: proatividade, capacidade de articular e comunicar suas necessidades, de guardar um apropriado nível de autonomia e de discutir os caminhos da relação ao longo do tempo, entre outros ${ }^{7}$. É importante também que o tutorando seja capaz de acreditar em seus tutores e respeitá-los, e, ao mesmo tempo, de desenvolver independência de julgamento e tomada de decisão ao longo do tempo para começar a se separar intelectualmente e emocionalmente do tutor.

\section{O papel do preceptor}

$\mathrm{Na}$ língua portuguesa preceptor significa o que dá preceitos ou instruções, mentor, mestre. Na área de saúde o preceptor é o profissional de nível superior, responsável pela integração teoria-prática num campo de estágio e/ou residência. Ensina, supervisiona, orienta e conduz o aluno na prática da futura profissão. Este profissional é o responsável pelo acompanhamento do processo de trabalho do residente bem como do treinamento em serviço. Suas atribuições são, entre elas, estabelecer cronograma de visitas a UBS, fazer a ponte entre os residentes e o serviço, facilitar o desenvolvimento das competências - conhecimento, habilidade e atitude - atribuídas ao residente, acompanhar quando possível os residentes nas atividades, nortear os residentes nas atividades de gerenciamento (planejamento, organização e avaliação) das atividades coletivas de saúde; avaliar criteriosamente e com prudência integralmente as ações exercidas pelo residente e dialogar sobre os pontos positivos e sugestões para aprimoramento; ministrar aulas na sua área de conhecimento e/ou participar das aulas de outros docentes, com a finalidade de adequar a teoria à prática; definir com o docente o conteúdo das aulas, visando à aplicação na prática, participar de reuniões com preceptores, tutores e coordenação. Enfim, perante esse arcabouço de responsabilidades, alguns dados corroboram que o preceptor precisa ser um protagonista nesse cenário, pois é o corresponsável da formação do residente e por isso é imprescindível que o mesmo tenha fundamentação científica e pedagógica para a construção /reconstrução do conhecimento ${ }^{12}$.

Os profissionais selecionados para exercer o papel de preceptor junto à coordenação da residência atuavam na assistência e apresentavam avaliação de desempenho positiva e tempo de experiência no PSF. Acreditou-se que a experiência prática desses profissionais favoreceria o processo de formação do residente. Porém, foi observado que em relação à parte acadêmico-científica estes profissionais tinham pouco conhecimento, e por se tratar de uma atividade complexa, era necessário conhecimento, sensibilidade, criatividade, improvisação e muito bom senso ${ }^{11}$.

A partir da análise dos discursos foi possível extrair as seguintes visões que o residente apresentava do preceptor.

"O papel dos preceptores era mais dar essa aula pra gente e tá sempre fazendo as visitas pra ver se tinha 
algum problema, eu tive amigo que teve problema com o tutor, então o papel do preceptor era isso" (sujeito 1).

“(...) os preceptores eram assim: eles acumulavam outras atividades, eu acho isso um problema, porque eles eram nossos preceptores, eles eram coordenadores, alguns atendiam então eles acumulavam várias outras atividades. Então assim, a gente tinha aulas com eles, acho que eles fizeram o máximo, dentro da capacidade deles, só que tem coisas que a gente reivindicava e não conseguia ser atingido, porque estava acima do que eles podiam fazer" (sujeito 4 ).

Os estudos sobre o papel educador do preceptor são raros e ainda há muito que se investigar sobre este ator nas práticas dos cursos de residência, mas segundo alguns estudiosos, o preceptor tem a função de estreitar a distância entre teoria e prática ${ }^{13,14}$.

É importante lembrarmos que os recém-formados chegam ao ambiente de trabalho com perfil generalista, para atuar com qualidade e resolutividade na atenção básica. No entanto, no início da carreira é comum o profissional se deparar com casos novos da rotina clínica, e embora a graduação os prepare para a tomada de decisão, o auxílio de um profissional experiente e capacitado é essencial para garantir o bom desempenho no serviço, portanto, o preceptor tem o papel de suporte, para ajudar o novo profissional a adquirir prática, até que este tenha maior confiança e segurança em suas atividades diárias.

Como foi relatado pelos egressos neste estudo, em muitos momentos os papéis de cada ator no processo de formação (residente, tutor, preceptor, equipe) não ficou muito claro. Portanto, caberia ao preceptor explicitar esses objetivos e aproximar a realidade teórica da realidade prática construindo um cenário favorável à aquisição de novos conhecimentos.

\section{CONSIDERAÇÕES FINAIS}

A apreciação dos documentos, a ausculta atenta e a análise dos discursos dos cirurgiões-dentistas egressos evidenciou que os tutores e preceptores desempenham papéis fundamentais na RMSF. Os tutores, no dia a dia do serviço, orientam o residente na sua formação e os preceptores, apesar de não possuírem um contato diário com o residente, exercem uma função indispensável na mediação das relações entre coordenação-residentetutor. É do preceptor também o papel de aproximação dos conteúdos teóricos com a prática do residente.

Foi possível concluir, pela análise dos discursos, que estes papéis não estavam bem definidos pelos atores envolvidos no processo de formação, o que dificultou o enfretamento de alguns problemas vivenciados pelos residentes na prática dos serviços. Torna-se evidente a necessidade de um melhor preparo destes profissionais para que as práticas pedagógicas operadas na residência sejam realizadas a contento.

Da mesma forma se evidencia a necessidade da institucionalização da formação de recursos humanos em saúde para atuar na residência multiprofissional, a fim de que se alcance o fortalecimento da proposta na atenção primária com vistas à promoção, prevenção e recuperação da saúde da população. Para tanto a academia deve reconhecer essa nova modalidade de pós-graduação e 
exercer seu papel formador, criando mecanismos que possibilitem ao profissional da rede de saúde condições de se aprimorar nas questões relacionadas à educação para saúde.

Certamente a educação continuada, por meio das modalidades de mestrados profissionais e cursos de especialização, voltados para saúde pública, são elementos chaves para avançarmos na qualidade da formação no serviço, pois articula os dois saberes de maneira bastante transparente e com objetivos definidos, ou seja, qualificar o profissional para o serviço no Sistema Único de Saúde, a fim de garantir subsídios visando a superação dos desafios impostos pelo complexo papel dos tutores e preceptores do serviço público.

\section{Agradecimentos}

Os autores agradecem à Organização Pan-Americana da Saúde (OPAS) e ao Ministério da Saúde (MS) pelo financiamento desta pesquisa, por meio do Observatório de Recursos Humanos em Odontologia.

\section{REFERÊNCIAS}

1. Starfield B. Atenção primária: equilíbrio entre necessidades de saúde, serviços e tecnologia. Brasília (DF): UNESCO; Ministério da Saúde; 2002.

2. Organização Mundial de Saúde. Primeira conferência internacional sobre promoção da saúde; 17-21 nov. 1986; Ottawa, Canadá.

3. da Motta L, Pacheco L. Integrating medical and health multiprofessional residency programs: the experience in building an interprofessional curriculum for health professionals in Brazil. Educ Health. 2014;27(1):83.
4. Faculdade de Medicina de Marília. Manual da residência multiprofissional em saúde da família. Marília: Faculdade de Medicina de Marília; 2005.

5. Bardin L. Análise de conteúdo. Lisboa: Edições 70, 2011.

6. Ross J, Head K, King L, Perry PM, Smith S. The personal development tutor role: An exploration of student and lecturer experiences and perceptions of that relationship. Nurse Educ Today. 2014;34(9):1207-13.

7. Bellodi PL, de Arruda Martins M. Tutoria: mentoring na formação médica. São Paulo: Casa do Psicólogo; 2005.

8. de Oliveira BottiI SH, RegoI S. Preceptor, supervisor, tutor e mentor: quais são seus papéis? Rev Bras Educ Med. 2008;32(3):363-73.

9. Moscatello ELM. A vivência do enfermeiro como tutor em um hospital de especialidades oncológicas [dissertação]. São Paulo: Universidade de São Paulo, Escola de Enfermagem, 2008.

10. Pinto LLS, Formigli VLA, Rêgo RdCF. A dor ea delícia de aprender com o SUS: integração ensino-serviço na percepção dos internos em medicina social. RBSP. 2007; 31(1):115-33.

11. Oliveira FR. Residência multiprofissional em saúde da família: limites e possibilidades para mudança na formação profissional [dissertação]. São Paulo: Pontifícia Universidade Católica; 2007.

12. Ribeiro KRB, do Prado ML. A prática educativa dos preceptores nas residências em saúde: um estudo de reflexão. RGE. 2014;35(1):161-5. 
13. Bain L. Preceptorship: a review of the literature. J Adv Nurs. 1996; 24(1):104-7.

14. Armitage P, Burnard P. Mentors or preceptors? Narrowing the theorypractice gap. Nurse Educ Today. 1991;11(3):225-9.

\section{ABSTRACT \\ The training of educators in family health: an agenda for future activities in Dentistry}

The multi-professional residency is a kind of graduate program that gathers health professionals and aims the consonance of the academic education with the public health needs that will take place in primary health care, especially through the Family Health Strategy. This study aims to analyze the perspective of the dental professionals coming from the multiprofessional residency, on the roles of two kinds of educators: tutors and supervisors. The qualitative approach study consists of two steps: document analysis of the multiprofessional residency course at Faculty Santa Marcelina of São Paulo and semistructured interviews with the dentists who graduated from that course. After this analysis it was possible to conclude that the roles of the tutors and supervisors was not clearly defined in the process of the residence academic education, thus making it difficult for them to perform their tasks. The need of a better preparation and definition of these professionals' role is essential for the proper performance of pedagogical practices. Likewise, the need of an Institutionalization of the Human Resources formation becomes evident, in a multi-professional health level, in the curricula of the higher education institutions, in order to enhance this proposal in the primary health care contributing with the promotion, prevention and recovery of people's health.

Descriptors: Residency. Family Health. Primary Health Care. Dentistry.

Correspondência para:

Profa. Dra. Fernanda Campos Sousa de Almeida

e-mail: fernandacsa@usp.br

Faculdade de Odontologia da Universidade de São Paulo

Av. Prof. Lineu Prestes, 2227

05558-000 Cidade Universitária - São Paulo/SP 